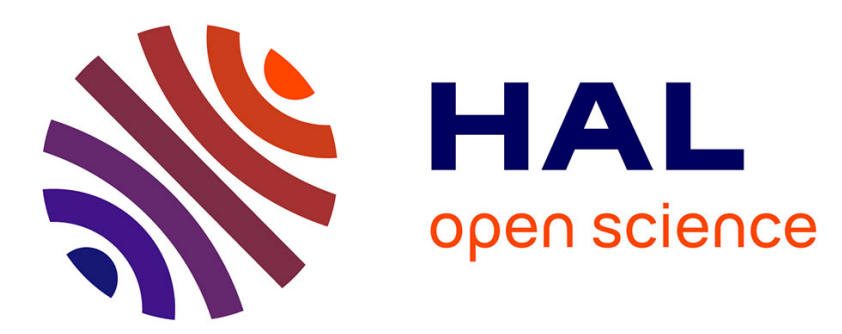

\title{
Analysis of a Sugimoto's model of nonlinear acoustics in an array of Helmholtz resonators
}

Stéphane Junca, Bruno Lombard

\section{To cite this version:}

Stéphane Junca, Bruno Lombard. Analysis of a Sugimoto's model of nonlinear acoustics in an array of Helmholtz resonators. SIAM Journal on Applied Mathematics, 2020, 80 (4), pp.1704-1722. 10.1137/19M1280624. hal-02186692v2

\section{HAL Id: hal-02186692 \\ https://hal.science/hal-02186692v2}

Submitted on 21 Apr 2020

HAL is a multi-disciplinary open access archive for the deposit and dissemination of scientific research documents, whether they are published or not. The documents may come from teaching and research institutions in France or abroad, or from public or private research centers.
L'archive ouverte pluridisciplinaire HAL, est destinée au dépôt et à la diffusion de documents scientifiques de niveau recherche, publiés ou non, émanant des établissements d'enseignement et de recherche français ou étrangers, des laboratoires publics ou privés. 


\title{
ANALYSIS OF A SUGIMOTO'S MODEL OF NONLINEAR ACOUSTICS IN AN ARRAY OF HELMHOLTZ RESONATORS
}

\author{
STÉPHANE JUNCA* AND BRUNO LOMBARD ${ }^{\dagger}$
}

\begin{abstract}
A coupled system involving a nonlinear scalar PDE and a linear ODE is theoretically investigated. This hypebolic system with relaxation models the propagation of nonlinear waves in a waveguide connected to Helmholtz resonators, this device being an example of a nonlinear acoustic metamaterial. In a previous paper [Sugimoto, J. Fluid. Mech. 1992], it has been shown that this device allows also the propagation of acoustic solitons. In the present paper, the mathematical properties of the coupled system are analysed: formation of singularity in finite time, existence of entropy solutions in fractional BV spaces and uniqueness with a single family of entropies. New results are also deduced about weakly coupled systems. Numerical simulations illustrate these findings.
\end{abstract}

Key words. nonlinear waves; hyperbolic systems; acoustic solitons; fractional BV spaces; balance laws; shocks

AMS subject classifications. 26A45, 35L65, 35L67, 76N10.

1. Introduction. In a series of papers [37, 38, 39, 40], Sugimoto and coauthors proposed a model to describe the propagation of nonlinear acoustic waves in a tube connected to a set of Helmholtz resonators (Fig. 1). The original motivation of this work was to describe the high-amplitude waves generated by high-speed trains in tunnels, and to propose resonators as a mean to counterbalance the effect of shock waves. The Sugimoto's model writes as a coupled one-dimensional system of PDE and $\operatorname{ODE}(x \in \mathbb{R}, t>0)$ :

$$
\left\{\begin{array}{l}
\partial_{t} u+\partial_{x}\left(a u+b \frac{u^{2}}{2}\right)=-\Omega^{2} \partial_{t} p, \\
\partial_{t}^{2} p+\varepsilon \partial_{t} p+\omega_{0}^{2} p=u
\end{array}\right.
$$

with the coefficients $a>0, b>0, \varepsilon \geq 0$, the characteristic frequencies $\omega_{0}$ and $\Omega$, and the initial data

$$
u(x, 0)=u_{0}(x), \quad p(x, 0)=p_{0}(x), \quad \partial_{t} p(x, 0)=p_{1}(x) .
$$

Subsequently, the Sugimoto's model has led to numerical, experimental and theoretical works $[25,35,31]$. Enrichment of this model, in particular by taking into account nonlinear attenuation mechanisms in (1.1b), have led to the first experimental observation of acoustic solitons [35].

Besides its physical relevance, the Sugimoto's model is of mathematical interest. It can be put in the form of a first-order $3 \times 3$ diagonal hyperbolic system. For systems of this form with $N$ equations, the existence of entropic solutions in $\mathrm{BV}$ is known by using $N=3$ families of entropies [32]. One objective here is to prove existence of solutions to (1.2) in fractional $\mathrm{BV}^{s}$ spaces. The latter have been introduced within the framework of homogeneous scalar conservation laws [5], and subsequently extended to the case of a $2 \times 2$ system issued from chemical engineering [6]. The structure of functions in $\mathrm{BV}^{s}$ is similar to that of $\mathrm{BV}$ with left and right traces. This aspect

\footnotetext{
*Université Côte d'Azur, INRIA \& CNRS, LJAD, Parc Valrose, 06108, Nice, France (junca@unice.fr).

$\dagger^{\dagger}$ Aix Marseille Univ, CNRS, Centrale Marseille, LMA UMR 7031, Marseille, France (lombard@lma.cnrs-mrs.fr).
} 
is fundamental for preserving the shock structure of the solutions. In addition, $\mathrm{BV}^{s}$ is larger than $\mathrm{BV}$. The regularity of functions in $\mathrm{BV}^{s}$ corresponds to the regularity of Sobolev in $W^{s, p}, s p<1$ [5]. An example of the application of $\mathrm{BV}^{s}$ spaces is to characterize the smoothing effect for entropy solutions of nonlinear scalar conservation laws $[5,11,29]$. These recent results respond in the one-dimensional case to a Lions, Perthame and Tadmor conjecture on fractional regularity in Sobolev spaces, and keep the properties on the traces for the entropy solutions given in [11].

Another objective is to analyze the formation of shock. The Burgers equation in the 1.h.s. of (1.1a) suggests the appearance of finite time shock. In counterpart, the source term in the r.h.s. can counterbalance nonlinearity and prevent the occurrence of singularity. Moreover, this source term is non-local in time (1.1b), which complicates the analysis. In the framework of weak entropy solutions, uniqueness results are then sought with only one family of entropies.

The article is organized as follows. Sec. 2 recalls the physical modeling underlying the system (1.1), which is rewritten as a first-order $3 \times 3$ diagonal hyperbolic system. In Sec. 3, the breakdown of regular solutions for large initial data is investigated (Theorems 3.1 and 3.2). Existence of global smooth solutions for small data is expected physically. Therefore it is studied near equilibrium, based on the ShizutaKawashima coupling condition [36]. Numerical experiments illustrate the discussion. In Sec. 4, Theorem 4.3 states the existence of a global weak entropy solution for initial data in $\mathrm{BV}^{s}$. Contrary to existing results [32], one family of entropies is sufficient to prove the uniqueness of the entropy solution. Sec. 5 makes a link with the theory of weakly coupled systems, for which existence and uniqueness results are already known [32]. Theorem 5.3 extends these results to the case of $\mathrm{BV}^{s}$ solutions. Lastly, future directions of research are outlined in Sec. 6 .

\section{Problem statement.}

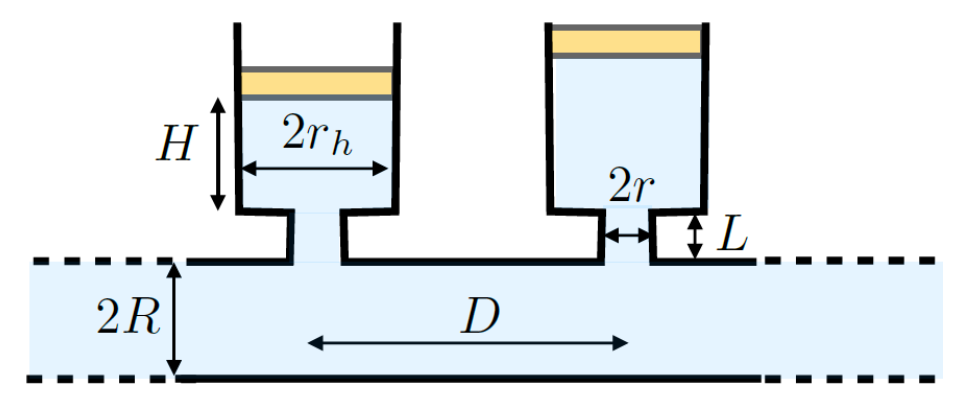

FIG. 1. sketch of the guide connected with Helmholtz resonators.

2.1. Sugimoto's model. Let us consider an air-filled tube connected with a network of Helmholtz resonators (Fig. 1). The cylindrical resonators are uniformly distributed along the tube. The geometrical parameters are the radius of the guide $R$, the axial spacing between resonators $D$, the radius of the neck $r$, the length of the neck $L$, the radius of the cavity $r_{h}$ and the height of the cavity $H$, which may vary depending on the resonator. Hence, the cross-sectional area of the guide is $A=\pi R^{2}$ and the volume of each resonator is $V=\pi r_{h}^{2} H$.

The physical parameters are the ratio of specific heats at constant pressure and volume $\gamma$, the pressure at equilibrium $p_{0}$, the density at equilibrium $\rho_{0}$, and a phenomenological term modeling the losses in the necks of the resonators $\epsilon$. The other 
dissipation mechanisms are neglected: the sound diffusivity is negligible, and the boundary layer in the tube involves fractional derivatives, which complicates the analysis. It yields the linear sound speed $a_{0}$ and the angular frequencies $\omega_{0}$ and $\Omega$ :

$$
a_{0}=\sqrt{\frac{\gamma p_{0}}{\rho_{0}}}, \quad \omega_{0}=a_{0} \sqrt{\frac{B}{L V}}=a_{0} \frac{r}{r_{h}} \frac{1}{\sqrt{L H}}, \quad \Omega=\sqrt{\frac{V}{2 \rho_{0} a_{0} A D}} .
$$

The following assumptions are made about the waves of angular frequency $\omega$ and wavelength $\lambda$ propagating in the tube:

- low-frequency regime $\left(\omega<\omega^{*} \approx \frac{1.84 a_{0}}{R}\right)$, so that only the plane mode propagates and the $1 \mathrm{D}$ approximation is valid [8];

- weak acoustic nonlinearity in the tube (small Mach number) [14];

- continuous distribution of resonators (wavelength $\lambda \gg D$ );

- linear response of the resonators, no turbulence.

Under these assumptions, the system can be described by a one-dimensional configuration with a surfacic distribution of resonators, where the unknowns are the axial velocity of the gas $u$ and the excess pressure in the cavity (compared to the guide) $p$. The right-going simple wave is then modeled by a coupled PDE-ODE system initially proposed by Sugimoto [37]:

$$
\left\{\begin{array}{l}
\partial_{t} u+\partial_{x}\left(a_{0} u+\frac{\gamma+1}{2} \frac{u^{2}}{2}\right)=-\Omega^{2} \partial_{t} p, \\
\partial_{t}^{2} p+\epsilon \partial_{t} p+\omega_{0}^{2} p=\omega_{0}^{2} \frac{\gamma p_{0}}{a_{0}} u
\end{array}\right.
$$

Defining the normalization parameter $\theta=\omega_{0}^{2} \frac{\gamma p_{0}}{a_{0}}$, the scaled coordinate $t \rightarrow t \sqrt{\theta}$ and the scaled positive parameters, which may vary with $x$ :

$$
a=\frac{a_{0}}{\sqrt{\theta}}, \quad b=\frac{\gamma+1}{2 \sqrt{\theta}}, \quad \varepsilon:=\frac{\epsilon}{\sqrt{\theta}}, \quad \omega_{0}:=\frac{\omega_{0}}{\sqrt{\theta}},
$$

then the system (2.2) recovers (1.1). It is noted that the system originally proposed incorporates additional terms of attenuation [37]. The full system is given in Sec. 6 .

2.2. First-order hyperbolic system. Introducing the new variable $\varphi:=\partial_{t} p$, the Sugimoto's system (1.1) is put in the form of a first-order system

$$
\left\{\begin{array}{l}
\partial_{t} u+\partial_{x}\left(a u+b \frac{u^{2}}{2}\right)=-\Omega^{2} \varphi \\
\partial_{t} \varphi=u-\varepsilon \varphi-\omega_{0}^{2} p \\
\partial_{t} p=\varphi
\end{array}\right.
$$

with initial data

$$
u(x, 0)=u_{0}(x), \quad \varphi(x, 0)=\varphi_{0}(x), \quad p(x, 0)=p_{0}(x) .
$$

Compatibility between these initial data and those of the $2 \times 2$ Sugimoto's system will be examined further in (3.3). Let us consider smooth solutions of (2.4). Multiplying (2.4a) by $u,(2.4 \mathrm{~b})$ by $\Omega^{2} \varphi$ and (2.4c) by $\Omega^{2} \omega_{0}^{2} p$, adding the terms and integrating with respect to $x$ yields

$$
\frac{d}{d t} \frac{1}{2} \int_{\mathbb{R}}\left(u^{2}+\Omega^{2} \varphi^{2}+\Omega^{2} \omega_{0}^{2} p^{2}\right) d x=-\int_{\mathbb{R}} \varepsilon \Omega^{2} \varphi^{2} d x .
$$


The energy in l.h.s. in (2.6) is convex. If $\varepsilon>0$, the system is dissipative, whereas $\varepsilon=0$ describes a conservative system. For weak solutions of (2.8), the equality in (2.6) becomes an inequality. Setting

$$
\mathbf{U}=(u, \varphi, p)^{\top}, \quad \mathbf{F}(\mathbf{U})=\left(a u+b \frac{u^{2}}{2}, 0,0\right)^{\top}, \quad \mathbf{S}=\left(\begin{array}{ccc}
0 & -\Omega^{2} & 0 \\
1 & -\varepsilon & -\omega_{0}^{2} \\
0 & 1 & 0
\end{array}\right),
$$

the system (2.4) writes as a balance laws

$$
\partial_{t} \mathbf{U}+\partial_{x} \mathbf{F}(\mathbf{U})=\mathbf{S} \mathbf{U} .
$$

The flux $\mathbf{F}$ in (2.7) is diagonal. The Jacobian matrix $\mathbf{A}(\mathbf{U})=\nabla_{\mathbf{U}} \mathbf{F}(\mathbf{U})$ has 2 real eigenvalues:

- the eigenvalue $a+b u$ is genuinely nonlinear with the eigenvector $e_{1}=(1,0,0)^{\top}$;

- the eigenvalue 0 is linearly degenerate of multiplicity 2 , with an eigenspace generated by $e_{2}=(0,1,0)^{\top}$ and $e_{3}=(0,0,1)^{\top}$.

Without the source term, the system is diagonal, hence $u, \varphi$ and $p$ are the Riemann invariants. The eigenvalues of $\mathbf{S}$ are

$$
\operatorname{Sp}(\mathbf{S})=\left\{0, \frac{1}{2}\left(-\varepsilon+\left(\varepsilon^{2}-4\left(\Omega^{2}+\omega_{0}^{2}\right)\right)^{1 / 2}\right), \frac{1}{2}\left(-\varepsilon-\left(\varepsilon^{2}-4\left(\Omega^{2}+\omega_{0}^{2}\right)\right)^{1 / 2}\right)\right\} .
$$

If $\varepsilon \neq 2 \sqrt{\Omega^{2}+\omega_{0}^{2}}$, then $\mathbf{S}$ is diagonalizable.

3. Formation of singularities. In the case of large data, we prove sufficient conditions for the breakdown of regular solutions in finite time. For this purpose, the $2 \times 2$ system (1.1) is first transformed into a scalar equation with a source term (Sec. 3.1). Then one considers successively the dissipative case $\varepsilon>0$ (Sec. 3.2) and the conservative case $\varepsilon=0$ (Sec. 3.3). In the case of small data, global smooth solutions are expected: the Sugimoto's model has been defined to prevent from the occurence of shocks and to propagate acoustic solitons [37]. The Shizuta-Kawashima is invocated to study the possible existence of global smooth solutions near equilibrium (Sec. 3.4). Numerical experiments are proposed to illustrate these properties (Sec. 3.5).

3.1. Burgers equation with memory. Equation (1.1b) is differentiated in terms of $t$. Using $\varphi=\partial_{t} p$ yields:

$$
\left\{\begin{array}{l}
\partial_{t} u+\partial_{x}\left(a u+b \frac{u^{2}}{2}\right)=-\Omega^{2} \varphi, \\
\frac{d^{2} \varphi}{d t^{2}}+\varepsilon \frac{d \varphi}{d t}+\omega_{0}^{2} \varphi=\partial_{t} u \equiv \sigma
\end{array}\right.
$$

with the initial data

$$
u(x, 0)=u_{0}(x), \quad \varphi(x, 0)=\varphi_{0}(x), \quad \frac{d \varphi}{d t}(x, 0)=\varphi_{1}(x) .
$$

Smooth solutions of (1.1) are solutions of (3.1), provided the initial data (3.2) satisfy the compatibility condition

$$
\varphi_{1}(x)=u_{0}(x)-\varepsilon \varphi_{0}(x)-\omega_{0}^{2} p_{0}(x)
$$


Conversely, integrating (3.1b) with respect to $t$ recovers (1.1) under the condition (3.3). The explicit solution of (3.1b) is

$$
\varphi(x, t)=\varphi_{0}(x) J_{0}(t)+\varphi_{1}(x) J_{1}(t)+\int_{0}^{t} J_{1}(t-s) \sigma(x, s) d s,
$$

where $J_{0}$ and $J_{1}$ depend on the coefficients of (3.1b). Setting

$$
\Delta^{2}=\varepsilon^{2}-\left(2 \omega_{0}\right)^{2}, \quad \delta=\sqrt{|\Delta|},
$$

calculations yield the following 4 cases:

- $\varepsilon=0, \omega_{0}=0$ :

$$
J_{0}(t)=1, \quad J_{1}(t)=t
$$

- $0 \leq \varepsilon<2 \omega_{0}$ :

$$
\begin{aligned}
& J_{0}(t)=\exp (-\varepsilon t / 2)\left(\cos (\delta t / 2)+\frac{\varepsilon}{\delta} \sin (\delta t / 2)\right) \underset{\varepsilon \rightarrow 0}{\equiv} \cos \omega_{0} t \\
& J_{1}(t)=\frac{2}{\delta} \exp (-\varepsilon t / 2) \sin (\delta t / 2) \underset{\varepsilon \rightarrow 0}{\equiv} \frac{1}{\omega_{0}} \sin \omega_{0} t .
\end{aligned}
$$

- $\varepsilon=2 \omega_{0}$ :

$$
J_{0}(t)=\left(1+\frac{\varepsilon}{2} t\right) \exp (-\varepsilon t / 2), \quad J_{1}(t)=t \exp (-\varepsilon t / 2) .
$$

- $\varepsilon>2 \omega_{0}$ :

$$
\begin{aligned}
& J_{0}(t)=\frac{1}{2 \delta}((\varepsilon+\delta) \exp (-(\varepsilon-\delta) t / 2)-(\varepsilon-\delta) \exp (-(\varepsilon+\delta) t / 2)), \\
& J_{1}(t)=\frac{1}{\delta}(\exp (-(\varepsilon-\delta) t / 2)-\exp (-(\varepsilon+\delta) t / 2))
\end{aligned}
$$

The solution (3.4) is injected into (3.1a). Integration by parts and the property $J_{1}(0)=0$ allow to transform the $2 \times 2$ system (1.1) into the nonlocal scalar PDE $(3.10)$

$$
\begin{aligned}
\partial_{t} u+\partial_{x}\left(a u+b \frac{u^{2}}{2}\right) & =\varphi_{0}(x) K_{0}(t)+\left(\varphi_{1}(x)-u_{0}(x)\right) K_{1}(t)+\mathcal{L} u(x, t), \\
& =\varphi_{0}(x)\left(K_{0}(t)-\varepsilon K_{1}(t)\right)-\omega_{0}^{2} p_{0}(x) K_{1}(t)+\mathcal{L} u(x, t)
\end{aligned}
$$

where the compatibility condition (3.3) has been used, and with

$$
\begin{aligned}
& K_{0}(t)=-\Omega^{2} J_{0}(t), \quad K_{1}(t)=-\Omega^{2} J_{1}(t), \quad \mathcal{K}(t)=-\Omega^{2} J_{1}^{\prime}(t), \\
& \mathcal{L} u(x, t)=\int_{0}^{t} \mathcal{K}(t-s) u(x, s) d s .
\end{aligned}
$$

In the case where the resonators are initially at rest $p_{0}(x)=0$ and $p_{1}(x)=0$, then (3.10) reduces to the scalar PDE with memory

$$
\partial_{t} u+\partial_{x}\left(a u+b \frac{u^{2}}{2}\right)=\int_{0}^{t} \mathcal{K}(t-s) u(x, s) d s .
$$

This is a Burgers equation with a non-local source term as in [7]. 
3.2. Dissipative case. Based on (3.10)-(3.11), some norms are introduced:

$$
\begin{aligned}
& C_{0}=\left\|\varphi_{0}^{\prime}\right\|_{\infty}\left\|K_{0}-\varepsilon K_{1}\right\|_{\infty}+\omega_{0}^{2}\left\|p_{0}^{\prime}\right\|_{\infty}\left\|K_{1}\right\|_{\infty}, \\
& C=\int_{0}^{+\infty}|\mathcal{K}(t)| d t, \\
& C_{\sharp}=\frac{C+\sqrt{C^{2}+4 b C_{0}}}{2 b} \geq C .
\end{aligned}
$$

In the dissipative case $\varepsilon>0, C$ in (3.13) can be computed analytically from (3.7)-(3.9) and (3.11). Setting $t^{*}=\frac{1}{\delta} \arctan \frac{\delta}{\varepsilon}$, one obtains:

$$
C= \begin{cases}\frac{2 \Omega^{2} \varepsilon}{\varepsilon^{2}+4 \delta^{2}}\left(-1+\frac{4 \sqrt{1+(\delta / \varepsilon)^{2}}}{1-\exp (-(\varepsilon \pi) /(2 \delta))} \exp \left(-\varepsilon t^{*} / 2\right)\right) & \text { if } 0<\varepsilon<2 \omega_{0}, \\ \frac{4 \Omega^{2}}{\varepsilon \exp (1)} & \text { if } \varepsilon=2 \omega_{0}, \\ \frac{4 \Omega^{2}}{\varepsilon+\delta}\left(\frac{\varepsilon-\delta}{\varepsilon+\delta}\right)^{\frac{\varepsilon-\delta}{2 \delta}} & \text { if } \varepsilon>2 \omega_{0} .\end{cases}
$$

Sufficient conditions are now stated for the formation of singularity with smooth initial data.

TheOREM 3.1 (Shock in finite time: case $\varepsilon>0$ ). Let us assume that the initial data $\left(u_{0}, \varphi_{0}, p_{0}\right)$ are smooth with compact support. If inf $u_{0}^{\prime}(x)<-C_{\sharp}$ and $\sup u_{0}^{\prime}(x) \leq$ $C_{\sharp}$, then a shock appears in finite time.

Proof. Following the idea of Lax [19], we deduce from (3.10) the Riccati equation satisfied by $U=\partial_{x} u$ with initial condition $U_{0}=u_{0}^{\prime}$ :

$$
\begin{aligned}
\partial_{t} U+(a+b u) \partial_{x} U+b U^{2} & =\varphi_{0}^{\prime}(x)\left(K_{0}(t)-\varepsilon K_{1}(t)\right)-\omega_{0}^{2} p_{0}^{\prime}(x) K_{1}(t)+\mathcal{L} U \\
& \equiv k(x, t)+\mathcal{L} U
\end{aligned}
$$

As long as the solution is smooth, equation (3.15) is rewritten along the characteristics:

$$
\frac{d}{d t} U=-b U^{2}+k(x, t)+\mathcal{L} U
$$

The integration is now along characteristic curves, so that the operator $\mathcal{L}$ is modified; nevertheless, the notation $\mathcal{L}$ is kept for simplicity. In the case of the homogeneous Burgers equation, the Riccati ODE is simply

$$
\frac{d}{d t} U=-b U^{2}
$$

which blows-up at time $T^{*}=-1 /(b U(0))$ if $U(0)<0$. When a linear dissipative source term $-\lambda u$ is added to the Burgers equation, a similar analysis yields

$$
\frac{d}{d t} U=-b U^{2}-\lambda U
$$

whose threshold is $\inf U_{0}<-\lambda / b$. The analysis of (3.16) is more complex due to the nonlocal source term $\mathcal{L} U$, which can accelerate or moderate the blow-up of $U$. Roughly speaking, the competition between the nonlinear part and the linear part 
favors a blow-up if $\frac{d}{d t} U<0$ is large enough. Let us prove it under the assumptions of the theorem. At any $x$, equation (3.16) and the notations (3.13) give

$$
\frac{d}{d t} U \leq-b U^{2}+C_{0}+C \sup _{(y, s) \in \mathbb{R} \times[0, t]}|U(y, s)| .
$$

One introduces the suprema $m(t)=\inf U(., t)=U\left(x_{0}, t_{0}\right)$ and $M(t)=\sup U(., t)$. The existence of $x_{0}$ is ensured by the fact that the solution of (3.16) remains with compact support at all time (Sec. 4.2). If $m<-C_{\sharp}$, the inequality $-b m^{2}+C_{0}+$ $C|m|<0$ holds. Similarly, $M>C_{\sharp}$ implies $-b M^{2}+C_{0}+C|M|<0$. Under the two conditions

$$
m\left(t_{0}\right)<-C \sharp, \quad\left|m\left(t_{0}\right)\right|>M\left(t_{0}\right),
$$

it follows that the r.h.s of (3.17) is negative at $\left(x_{0}, t_{0}\right)$. Similarly, $|M|>C_{\sharp}$ implies that $M(t)<C_{\sharp}$ for all time: $C_{\sharp}$ is a barrier for $M(t)[16]$.

It remains to prove (3.18) for all $t$. These two conditions are valid at $t_{0}=0$ from the assumptions of the theorem. Assuming that (3.18) is true at a given $t_{0}, U\left(x_{0}, t\right)$ is decreasing at least locally for $t<t_{0}$ and $t \simeq t_{0}$. Thus, for $h>0$ small enough,

$$
\begin{aligned}
m\left(t_{0}+h\right) & \leq U\left(x_{0}, t_{0}+h\right)=U\left(x_{0}, t_{0}\right)+h \dot{U}\left(x_{0}, t_{0}\right)+\mathcal{O}\left(h^{2}\right), \\
& \leq m\left(t_{0}\right)+h \dot{U}\left(x_{0}, t_{0}\right)+\mathcal{O}\left(h^{2}\right), \\
& \leq m\left(t_{0}\right)+h\left(-b m\left(t_{0}\right)^{2}+C_{0}+C\left|m\left(t_{0}\right)\right|\right)+\mathcal{O}\left(h^{2}\right) .
\end{aligned}
$$

Denoting $\dot{m}$ the generalized right derivative of $m[9]$ :

$$
\dot{m}(t)=\limsup _{h>0, h \rightarrow 0} \frac{m(t+h)-m(t)}{h}
$$

then $m$ satisfies

$$
\dot{m}\left(t_{0}\right) \leq-b m\left(t_{0}\right)^{2}+C_{0}+C\left|m\left(t_{0}\right)\right| .
$$

It follows $m(t) \leq y(t)$, where $y$ satisfies

$$
\left\{\begin{array}{l}
\dot{y}=-b y^{2}+C_{0}-C y, \\
y(0)=y_{0}:=m(0)<-C_{\sharp} .
\end{array}\right.
$$

The solution of (3.22) is explicitly known and blows-up in finite time $T^{*}$ :

$$
T^{*}=-\frac{-\ln \alpha}{\sqrt{C^{2}+4 b C_{0}}}>0, \quad \alpha=\frac{C+\sqrt{C^{2}+4 b C_{0}}+2 b y_{0}}{C-\sqrt{C^{2}+4 b C_{0}}+2 b y_{0}},
$$

with $0<\alpha<1$. Since $y$ is an upper solution of $m$, it follows that $m$ blows up in finite time. The lifetime of $U=\partial_{x} u$ is smaller or equal than $T^{*}$.

In the case of the scalar Burgers equation with linear dissipation

$$
\left\{\begin{array}{l}
\partial_{t} u+\partial_{x}\left(a u+b \frac{u^{2}}{2}\right)=-\varepsilon u, \\
u(x, 0)=u_{0}(x)
\end{array}\right.
$$

shock occurs iff inf $u_{0}^{\prime}(x)<-\varepsilon / b \equiv C_{\sharp}$. This minimal slope of the initial data increases linearly with the dissipation parameter. In the case of Sugimoto's system, on the contrary, the shock limit $C_{\sharp}$ in (3.14) does not evolve monotonically with $\varepsilon$. It is recalled, nevertheless, that it is only a sufficient condition (and not necessary). 
3.3. Conservative case. Theorem 3.1 is now extended to the conservative case $\varepsilon=0$ with $\omega_{0} \neq 0$. Any $t>0$ can be written $t=n \pi /\left(2 \omega_{0}\right)+r$, with $0 \leq r<\pi /\left(2 \omega_{0}\right)$. From (3.7) and (3.11), one obtains

$$
\int_{0}^{t}|\mathcal{K}(\tau)| d \tau=\frac{2 \Omega^{2}}{\pi}\left(t-r+\frac{\pi}{2 \omega_{0}} \sin \omega_{0} r\right)
$$

and hence

$$
\|\mathcal{L}(., t)\|_{\infty} \leq C(1+t), \quad \text { with } C=\frac{2 \Omega^{2}}{\pi} \max \left(1, \frac{\pi}{2 \omega_{0}}\right) .
$$

THEOREM 3.2 (Shock in finite time: case $\varepsilon=0$ ). Let consider smooth initial data $\left(u_{0}, \varphi_{0}, p_{0}\right)$ with compact support. There exist two constants $C^{ \pm}>0$ depending on $\left(\varphi_{0}, p_{0}\right)$ such that if inf $u_{0}^{\prime}<-C^{-}$and $\sup u_{0}^{\prime} \leq C^{+}$, then a shock appears in finite time.

Proof. We follow the proof of Theorem 3.1 with the same notations. The main modification is that $C$ has to be replaced by $C t$ in (3.22) since the norm of the linear operator is now of order $t$, as seen in (3.25):

$$
\dot{y}=-b y^{2}+C_{0}-C(1+t) y .
$$

Contrary to the dissipative case, the ODE (3.26) has not separable variables yielding an explicit solution. To prove the theorem, it suffices to get an upper solution blowing up in finite time $T^{*}<1$. Setting $D=\max \left(C_{0}, 2 C\right)$, these solutions satisfy $y<0$ and

$$
\dot{y} \leq f(y):=-b y^{2}+D(1+|y|) .
$$

Let $z$ be the solution of $\dot{z}=f(z)$ and $z^{-}$be the negative root of $f(z)=0$. If $z_{0}<z^{-}$, then $z$ is an upper solution of $y$; it is also decreasing and its lifespan is $T\left(z_{0}\right)=\int_{z_{0}}^{+\infty} \frac{d z}{-b z^{2}+D(1+|z|)} \rightarrow 0$ when $z_{0} \rightarrow-\infty$. Thus, there exists a constant $C^{-}$ such that for $z_{0} \leq-C^{-}$, then $T\left(z_{0}\right)<1$. Consequently, the solution $y$ of $(3.26)$ with $y_{0} \leq-C^{-}$blows up before time 1 . The constant $C^{+}$is chosen as in the proof of Theorem 3.1 to keep the r.h.s. of (3.26) negative, which concludes the proof.

3.4. Case of small solutions. If $\varepsilon \neq 0$, the hyperbolic system (2.4) is dissipative. In this case, Theorem 3.1 gives sufficient conditions of singularity formation for large smooth initial data. But due to the dissipation, global smooth solutions are also expected if the initial data are smooth and sufficiently small.

To prove the existence of global smooth solutions near equilibrium, a sufficient condition is given in [15] based on two properties: the Shizuta-Kawashima condition (S-K) at the equilibrium, and a special form of the dissipation.

The S-K condition relates on the linearized equations at the equilibrium. It is optimal in the linear case to get asymptotic stability of the equilibrium, but only sufficient in the nonlinear case $[1,30]$. It is also possible that the $\mathrm{S}-\mathrm{K}$ condition is satisfied on a continuum set of equilibria except at one point [1] whithout affecting the conclusion of [15]. For the Sugimoto's model, the S-K condition is fulfilled at all equilibrium states except one, as proven in the proposition 3.3 below.

Proposition 3.3. The Shizuta-Kawashima condition is fulfilled at all non-zero equilibrium parametrized by $p_{e} \neq 0$ :

$$
\left(u_{e}, \varphi_{e}, p_{e}\right)^{\top}=\left(\omega_{0}^{2} p_{e}, 0, p_{e}\right)^{\top} .
$$


On the contrary, the Shizuta-Kawashima condition is not satisfied at rest:

$$
\left(u_{e}, \varphi_{e}, p_{e}\right)^{\top}=(0,0,0)^{\top} .
$$

Proof. When $u_{e} \neq 0, \operatorname{ker}(\mathbf{S})=\left\{s\left(\omega_{0}^{2}, 0,1\right)^{\top}, s \in \mathbb{R}\right\}$ is a one-dimensional subspace defined by $\varphi=0, u=\omega_{0}^{2} p$. The first eigenvector of $\mathbf{A}$ associated with the non-null eigenvalue, $e_{1}=(1,0,0) \notin \operatorname{ker}(\mathbf{S})$. The two-dimensional eigenspace associated to the eigenvalue 0 of $\mathbf{A}$ clearly does not belong to the kernel of $\mathbf{S}$.

On the contrary, if $u_{e}=0$, the Jacobian matrix writes $\mathbf{A}=a \mathbf{I}$ where $\mathbf{I}$ is the $3 \times 3$ identity matrix. The eigenspace of $\mathbf{A}$ encompasses the full space, so the ShizutaKawashima condition is not satisfied by (3.29).

The sufficient form of the dissipation has to be strictly entropy dissipative (definition 2 in [15]). Unfortunately, the Sugimoto model is only entropy dissipative (definition 1 in [15]). Thus, we cannot prove the existence of global smooth solutions [15]. Nevertheless, numerical experiments and the dissipative structure of the model suggest that smooth solutions could exist, even if it remains an open problem.

\begin{tabular}{llllll}
\hline$R(\mathrm{~m})$ & $D(\mathrm{~m})$ & $r(\mathrm{~m})$ & $L(\mathrm{~m})$ & $r_{h}(\mathrm{~m})$ & $H(\mathrm{~m})$ \\
0.025 & 0.1 & 0.01 & 0.02 & 0.0215 & 0.1 \\
\hline$\gamma$ & $p_{0}(\mathrm{~Pa})$ & $\rho_{0}\left(\mathrm{~kg} / \mathrm{m}^{3}\right)$ & $\epsilon\left(s^{-1}\right)$ & & \\
1.403 & $1.0110^{5}$ & 1.177 & 5000 & & \\
\hline$a$ & $b$ & $\varepsilon$ & $\Omega$ & $\omega_{0}$ & \\
$4.7510^{-3}$ & $1.6410^{-5}$ & $6.8410^{-2}$ & $3.0010^{-2}$ & $4.9410^{-2}$ & \\
\hline \multicolumn{5}{r}{ TABLE 1 }
\end{tabular}

Geometrical, physical and scaled parameters in Sugimoto's model (2.4).

\subsection{Numerical experiments.}

Numerical methods. The numerical resolution of (2.8) is detailed in [25]. It relies on a splitting strategy. The non-homogeneous system (2.8) is split into a homogeneous hyperbolic PDE

$$
\partial_{t} \mathbf{U}+\partial_{x} \mathbf{F}(\mathbf{U})=\mathbf{0}
$$

which is solved by a finite-volume scheme with flux limiters, and a linear ODE

$$
\partial_{t} \mathbf{U}=\mathbf{S} \mathbf{U}
$$

which is solved exactly. The coupling between the successive resolutions is done by the second-order Strang splitting [21]. The choice of the numerical set-up (mesh size $\Delta x$, variable time step $\Delta t$ ) ensure reference solutions, not polluted by numerical artefacts. The numerical value of a $\phi\left(x_{i}=i \Delta x, t_{n}=t_{n-1}+\Delta t\right)$ is denoted $\phi_{i}^{n}$.

To detect the emergence of a shock in the conservative case $\varepsilon=0$, we use the fact that energy in (2.6) is conserved in the case of smooth solutions. For this purpose, we define a discrete counterpart of the energy in (2.6):

$$
\mathcal{E}^{n}=\sqrt{\frac{\Delta x}{2} \sum_{i}\left(\left(u_{i}^{n}\right)^{2}+\Omega^{2}\left(\varphi_{i}^{n}\right)^{2}+\Omega^{2} \omega_{0}^{2}\left(p_{i}^{n}\right)^{2}\right)} .
$$

As long as the solution is smooth, $\mathcal{E}^{n}$ remains constant. When a shock emerges, then $\mathcal{E}^{n}$ decreases. Obviously, this numerical criterion is valid when the numerical dissipation of the numerical scheme is sufficiently small, which will be the case here. 


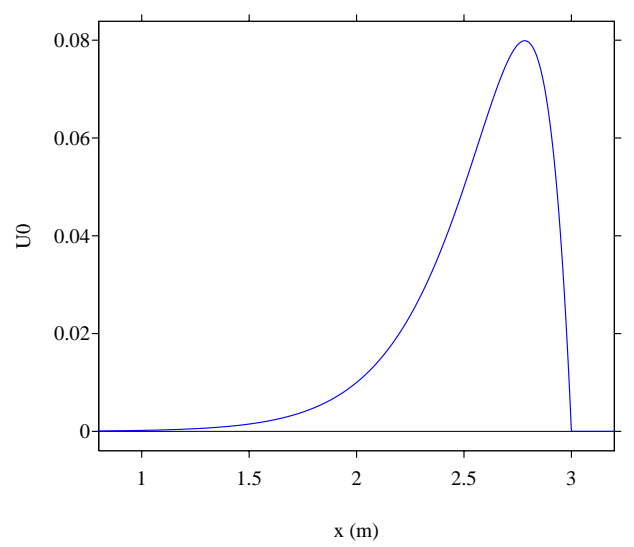

FIG. 2. Initial data $u_{0}(x)$ given in (3.31).
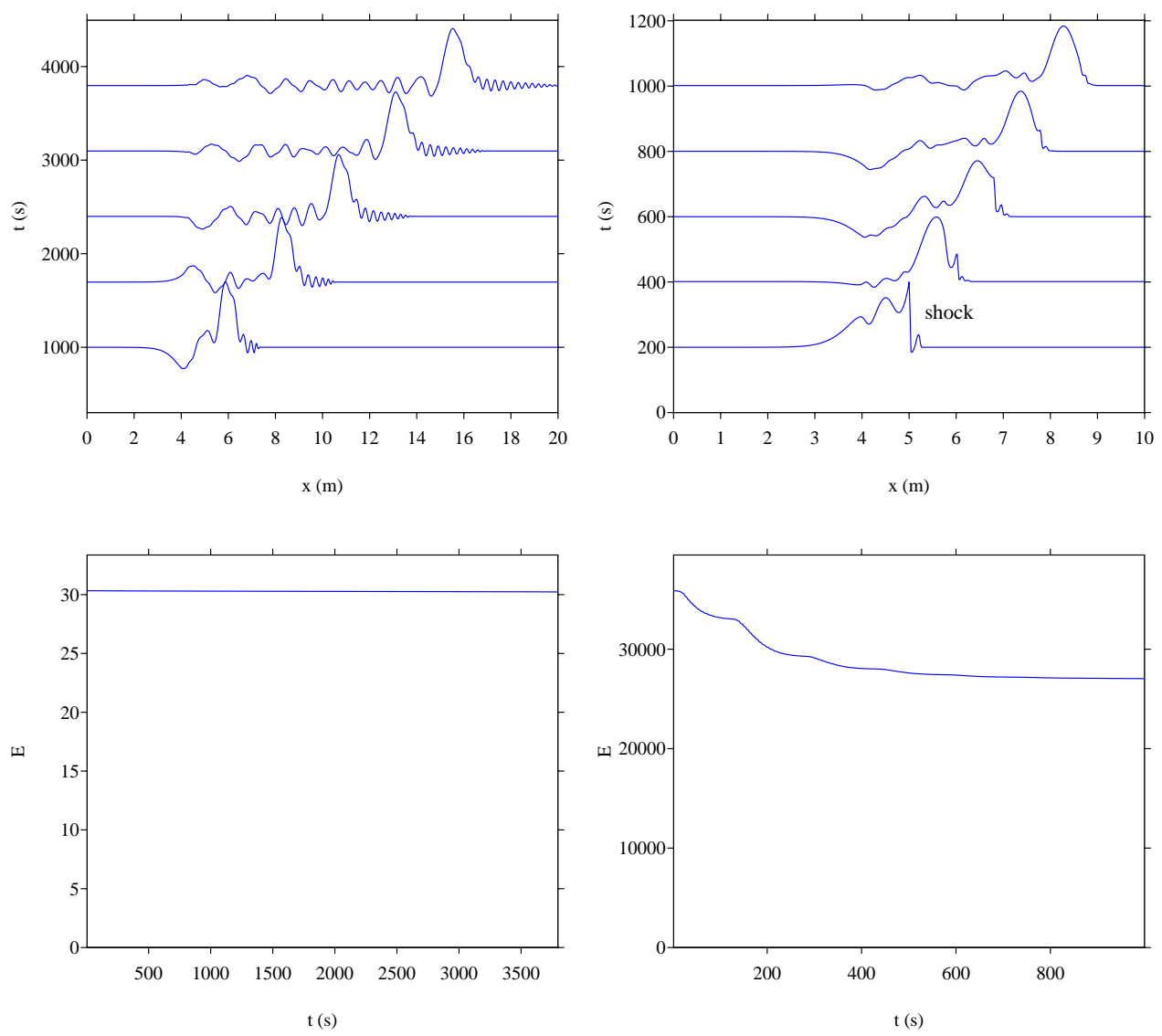

FIG. 3. Snapshots of $u$ at various instants (top) and time evolution of the discrete energy (bottom) in the conservative case $\varepsilon=0$. Left row: $A=100$, right row: $A=5000$.

Configuration. The numerical values of the geometrical and physical parameters (Sec. 2.1) are issued from [35]. Based on (2.3), il follows the scaled parameters (2.4). 
The full set of parameters is given in Table 1. In Theorem 3.1, the sufficient condition for the formation of shock involves different bounds for the maximum and minimum slopes. To place ourselves in the hypotheses of the theorem, we use as initial data

$$
u_{0}(x)=\left\{\begin{array}{ll}
g\left(x-x_{0}\right) & \text { if } x<x_{0}, \\
0 & \text { else, }
\end{array} \quad \text { with } g(x)=A x \exp (-x / \sigma) .\right.
$$

The other initial conditions are null: $\varphi_{0}=p_{0} \equiv 0$. In (3.31), one chooses $x_{0}=4$ $\mathrm{m}$ and $\sigma=0.427 \mathrm{~m}$, which corresponds roughly to a wavelength $\lambda=4 \mathrm{~m}$ (Fig. 2). The maximal and minimal slopes of the initial data (3.31) are deduced: $\sup u_{0}^{\prime}(x)=$ $u_{0}^{\prime}\left(x_{0}-2 \sigma\right)=A /(\exp (1))^{2}$ and $\inf u_{0}^{\prime}(x)=u_{0}^{\prime}\left(x_{0}\right)=-A$.

Results. Fig. 3 illustrates the conservative case $\varepsilon=0$, for which no bounds are explicitly known about the slopes $\inf u_{0}^{\prime}(x)$ and $\sup u_{0}^{\prime}(x)$ (Theorem 3.2). We represent $u$ at different instants as well as the temporal evolution of the discrete energy (3.30), for two values of the amplitude: $A=100$ and $A=5000$. If $A=100$, the discrete energy $\mathcal{E}$ is constant, which indicates that no shock appears. If $A=5000$ on the contrary, the discrete energy drops from the first moments, then reaches a plateau. It indicates the formation of a shock visible in at $t=200 \mathrm{~s}$. Once the shock is formed, a smooth soliton emerges and propagates without deformation.
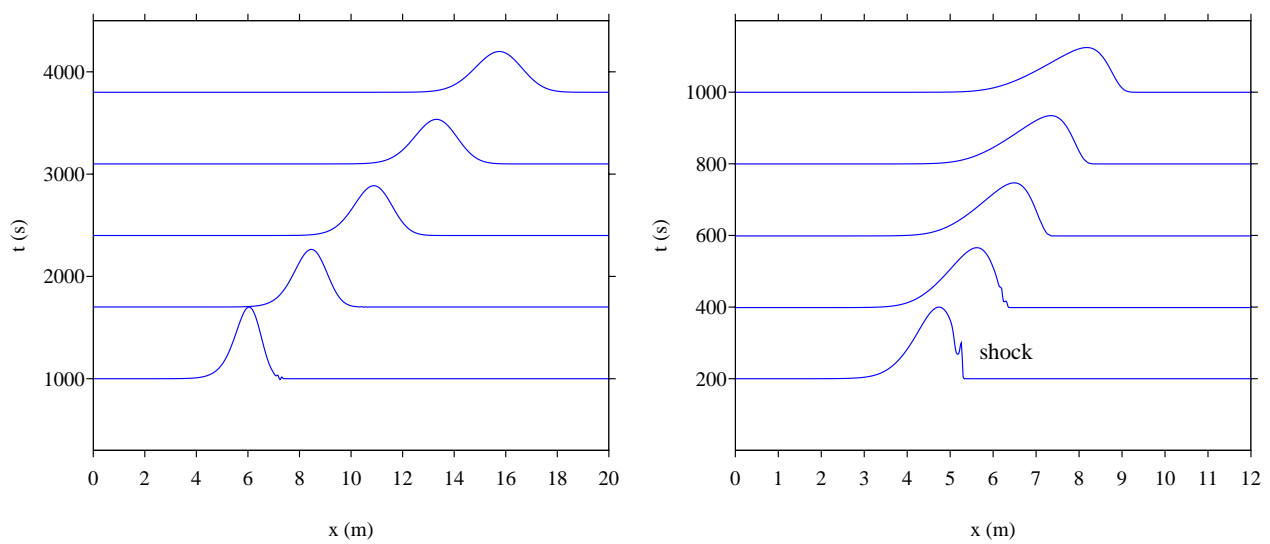

FIG. 4. Snapshots of $u$ at various instants in the dissipative case $\varepsilon \neq 0$. Left row: $A=100$, right row: $A=5000$.

The dissipative case is illustrated in Fig. 4. The discrete energy is no longer shown, since it decreases independantly from the occurence of shocks, and thus it does not give an indicator of singularity formation. The initial data yield $C_{0}=0$ and $C_{\sharp}=1215.55$ in (3.13). If $A=100$, then $\sup u_{0}^{\prime}(x)=13.53<C_{\sharp}$ but inf $u_{0}^{\prime}(x)=$ $-100>C_{\sharp}$ : the sufficient condition of singularity formation is no more satisfied. No shock is observed (left row). If $A=5000$, on the contrary, then $\sup u_{0}^{\prime}(x)=676<C_{\sharp}$ and $\inf u_{0}^{\prime}(x)=-5000<C_{\sharp}$ : the assumptions of Theorem 3.1 are satisfied. Indeed, a shock is observed during the first instants (right row).

\section{Large global $\mathrm{BV}^{s}$ solutions.}

4.1. Definitions and main result. Since shocks can occur, weak solutions are now considered.

DEFINITION 4.1 (Weak entropy solutions). Let consider the system (2.8) with initial data $\mathbf{U}_{0}$. A function $\mathbf{U} \in L_{l o c}^{\infty}\left(\left[0,+\infty\left[\times \mathbb{R}, \mathbb{R}^{3}\right)\right.\right.$ is a weak solution if, for all 
smooth vectorial test functions $\boldsymbol{\Phi} \in C_{c}^{\infty}\left(\mathbb{R} \times\left[0,+\infty\left[, \mathbb{R}^{3}\right)\right.\right.$ with compact support in $\mathbb{R} \times[0,+\infty[$, one has:

$$
\int_{0}^{+\infty} \int_{\mathbb{R}}\left(\mathbf{U} \cdot \partial_{t} \boldsymbol{\Phi}+\mathbf{F}(\mathbf{U}) \cdot \partial_{x} \boldsymbol{\Phi}+\boldsymbol{\Phi}^{\top} \cdot \mathbf{S} \mathbf{U}\right) d x d t+\int_{\mathbb{R}} \mathbf{U}_{0}(x) \cdot \boldsymbol{\Phi}(x, 0) d x=0 .
$$

Moreover, if $\mathbf{U} \in C^{0}\left(\left[0,+\infty\left[, L_{l o c}^{1}\left(\mathbb{R}, \mathbb{R}^{3}\right)\right.\right.\right.$ and satisfies for all convex entropy $\eta \in$ $C^{2}(\mathbb{R}, \mathbb{R})$ with the associated entropy flux $\psi^{\prime}(u)=(a+b u) \eta^{\prime}(u)$ and for all nonnegative scalar test functions $\phi \in C_{c}^{\infty}\left(\mathbb{R} \times\left[0,+\infty\left[, \mathbb{R}^{+}\right)\right.\right.$:

$$
\int_{0}^{+\infty} \int_{\mathbb{R}}\left(\eta(u) \partial_{t} \phi+\psi(u) \partial_{x} \phi+\eta^{\prime}(u) \Omega^{2} \varphi \phi\right) d x d t+\int_{\mathbb{R}} \eta\left(u_{0}(x)\right) \phi(x, 0) d x \geq 0,
$$

then $\mathbf{U}$ is called an entropy solution.

For weakly coupled systems, entropies are usually tested on each equation to get uniqueness results [32], as recalled in the forthcoming Sec. 5. On the contrary, the entropy condition in Definition 4.1 is only tested on the first equation (2.4a) of the Sugimoto's system. This choice is motivated by the fact that the only nonlinearity (generating shock waves) occurs in the first equation. As seen further, using a single family of entropies suffices to prove the uniqueness of the solution to (2.4). The fractional spaces $\mathrm{BV}^{s}, 0<s<1$, are recalled:

Definition $4.2\left(\mathrm{BV}^{s}\right.$ function). Let $T V^{s}$ be the total variation, also called the $p-$ variation with $p=1 / s$ :

$$
T V^{s}(u)=\sup _{n \in \mathbb{N},} \sum_{x_{0}<x_{1}<\ldots<x_{n}} \sum_{0 \leq i<n}\left|u\left(x_{i+1}\right)-u\left(x_{i}\right)\right|^{1 / s} .
$$

The $B V^{s}$ scalar functions on $\mathbb{R}$ are defined by $B V^{s}(\mathbb{R})=\left\{u, T V^{s}(u)<+\infty\right\} . A$ vectorial function is in $B V^{s}$ if all its components belong to $B V^{s}$. Equivalently, the $T V^{s}$ variation can be taken with a vectorial norm instead of the absolute value.

Now we state the main theorem of this paper, concerning the existence of $\mathrm{BV}^{s}$ solutions of the Sugimoto's system.

Theorem 4.3 (Large $\mathrm{BV}^{s}$ solutions). Let the initial data $\mathbf{U}_{0}=\left(u_{0}, \varphi_{0}, p_{0}\right)^{\top}$ belong to $B V^{s}\left(\mathbb{R}, \mathbb{R}^{3}\right), 0<s<1$. Then the system (2.4) admits an unique global weak entropy solution in

$$
L_{l o c}^{\infty}\left(\left[0,+\infty\left[, B V^{s}\left(\mathbb{R}, \mathbb{R}^{3}\right) \cap C_{l o c}^{s}\left(\left[0,+\infty\left[, L_{l o c}^{1 / s}\left(\mathbb{R}, \mathbb{R}^{3}\right) .\right.\right.\right.\right.\right.\right.
$$

Moreover, the solution satisfies the energy inequality:

$$
\frac{d}{d t} \frac{1}{2} \int_{\mathbb{R}}\left(u^{2}+\Omega^{2} \varphi^{2}+\Omega^{2} \omega_{0}^{2} p^{2}\right) d x \leq-\int \varepsilon \Omega^{2} \varphi^{2} d x
$$

REMARK 4.1 (BV solutions). For $s=1$, the usual $B V=B V^{1}$ solutions for scalar conservation laws are recovered by the Sugimotos' system where $C^{s}$ has to be replaced by Lipschitz: if $\mathbf{U}_{0}$ belongs to $B V\left(\mathbb{R}, \mathbb{R}^{3}\right)$ then

$$
\mathbf{U} \in L_{l o c}^{\infty}\left(\left[0,+\infty\left[, B V\left(\mathbb{R}, \mathbb{R}^{3}\right) \cap \operatorname{Lip}_{l o c}\left(\left[0,+\infty\left[, L_{l o c}^{1}\left(\mathbb{R}, \mathbb{R}^{3}\right) .\right.\right.\right.\right.\right.\right.
$$

REMARK 4.2 ( $L^{\infty}$ solutions). For $s=0$, the functional space is $B V^{0}=L^{\infty}$. For weakly coupled systems with initial data in $L^{\infty}$, results similar to 4.3 are already 
known [32], as seen in Sec. 5. In this particular case, Theorem 4.3 yields a new result: one whole family of scalar entropy $\eta \in C^{2}(\mathbb{R}, \mathbb{R})$ depending only on $u$ is sufficient to prove uniqueness.

The uniqueness of the entropy solution with only one entropy is well known for the Burgers' equation. Since only one field is nonlinear in Sugimoto's model, the result stated in Theorem 4.3 is not surprising. The rest of Sec. 4 is devoted to the proof of Theorem 4.3, which amounts to prove the propagation of the $\mathrm{BV}^{s}$ regularity in space for all positive time. The existence of $\mathrm{BV}^{s}$ solutions relies on a splitting scheme. The uniqueness follows from the classical Kruzkov proof of doubling of variables. Such a result is known for weakly coupled systems using as many families of entropy as the size of the system [32]; we check below that one single family of entropies is sufficient for the Sugimoto's system, which constitutes a slight improvement. Finally, it ends by the proof of the energy inequality.

4.2. Splitting scheme. The $\mathrm{BV}^{s}, \mathrm{BV}$ and $L^{\infty}$ estimates are obtained by a splitting scheme which splits the system (2.8) in two parts: first, the Burgers equation, second the differential systems. These two parts are studied independently.

Let $\Delta t>0, \mathbf{U}^{0}(x)=\mathbf{U}_{0}(x), \mathbf{U}^{n}=\left(u^{n}, \varphi^{n}, p^{n}\right)^{\top}$ stands for the approximate solution built by the splitting scheme at time $t_{n}=n \Delta t$. The half-step value $\mathbf{U}^{n+1 / 2}$ is the solution of the homogeneous hyperbolic PDE

$$
\left\{\begin{array}{l}
\left.\left.\partial_{t} \mathbf{U}+\partial_{x} \mathbf{F}(\mathbf{U})=\mathbf{0}, \quad(x, t) \in \mathbb{R} \times\right] t_{n}, t_{n+1}\right], \\
\mathbf{U}\left(x, t_{n}\right)=\mathbf{U}^{n}
\end{array}\right.
$$

Then $\mathbf{U}^{n+1}$ is given by the solution of the ODE parametrized by $x$,

$$
\left\{\begin{array}{l}
\left.\left.\frac{d}{d t} \mathbf{U}=\mathbf{S ~ U}, \quad t \in\right] t_{n}, t_{n+1}\right] \\
\mathbf{U}\left(., t_{n}\right)=\mathbf{U}^{n+1 / 2}
\end{array}\right.
$$

The splitting scheme can be performed in various ways, for instance a wave front tracking for Burgers [10] and an approximate solution for the ODE. We choose here the most precise splitting scheme. The hyperbolic part is only a Burgers equation which is solved exactly. The linear ODE is solved explicitly:

1. $u^{n+1 / 2}$ is the exact solution at time $t_{n}+\Delta t$ of the Burgers equation with initial data $u^{n}$. The other components are constant: $\varphi^{n+1 / 2}=\varphi^{n}$ and $p^{n+1 / 2}=p^{n}$; 2. $\mathbf{U}^{n+1}=\exp (t \mathbf{S}) \mathbf{U}^{n+1 / 2}$ is the exact solution of the ODE.

Burgers equation. It is well known that both the $L^{\infty}$ norm and the total variation of the entropy solution $u$ of the Burgers equation are non increasing [10]. A similar result has been obtained recently concerning the fractional total variation: $T V^{s}(u)$ is non increasing with respect to time [5].

Differential system. The ODE (4.6) writes

$$
\left\{\begin{array}{l}
\frac{d u}{d t}=-\Omega^{2} \varphi \\
\frac{d \varphi}{d t}=u-\varepsilon \varphi-\omega_{0}^{2} p, \\
\frac{d p}{d t}=\varphi .
\end{array}\right.
$$


The Euclidean norm associated to this differential system is

$$
|\mathbf{U}|_{2}^{2}=u^{2}+\Omega^{2} \varphi^{2}+\Omega^{2} \omega_{0}^{2} p^{2} .
$$

The $L^{\infty}$ norm and the total variation w.r.t. $x$ are chosen in accordance with (4.8):

$$
\begin{aligned}
& \|\mathbf{U}(x)\|_{2, \infty}=\sup _{x \in \mathbb{R}}|\mathbf{U}(x)|_{2}, \\
& T V_{2}^{s}(\mathbf{U})=\sup _{n \in \mathbb{N}, x_{0}<\ldots<x_{n}} \sum_{0 \leq i<n}\left|\mathbf{U}\left(x_{i+1}\right)-\mathbf{U}\left(x_{i}\right)\right|_{2}^{1 / s} .
\end{aligned}
$$

Proposition 4.4 (Decaying energy and total variation). The solution of (4.6) satisfies

$$
\left\{\begin{array}{l}
\frac{1}{2} \frac{d}{d t}|\mathbf{U}|_{2}^{2}=-\varepsilon \Omega^{2} \varphi^{2} \leq 0, \\
T V_{2}^{s}(\mathbf{U}(., t)) \leq T V_{2}^{s}(\mathbf{U}(., 0)) .
\end{array}\right.
$$

Proof. The derivation of (4.10a) is usual. It follows the same computation as for the global space energy decay (2.6), except there is no space variable. Mutiplying (4.7a) by $u,(4.7 \mathrm{~b})$ by $\Omega^{2} \varphi,(4.7 \mathrm{c})$ by $\Omega^{2} \omega_{0}^{2} p$ and adding all the terms yields

$$
\frac{1}{2} \frac{d}{d t}\left(u^{2}+\Omega^{2} \varphi^{2}+\Omega^{2} \omega_{0}^{2} p^{2}\right)=-\varepsilon \Omega^{2} \varphi^{2} .
$$

To study the total variation in space, let $x<y$. The differential system is linear with respect to the initial data $\mathbf{U}(x, 0)-\mathbf{U}(y, 0)$, thus $\mathbf{U}(x, t)-\mathbf{U}(y, t)$ satisfies also (4.6). Thus, the inequality $|\mathbf{U}(x, t)-\mathbf{U}(y, t)|_{2} \leq|\mathbf{U}(x, 0)-\mathbf{U}(y, 0)|_{2}$ is a direct consequence of the pointwise decay of the energy (4.10a). Summing up the variation and taking the supremum yields (4.10b).

Concerning the asymptotic behavior of the solution $\mathbf{U}(t)$ of the differential system (4.7) with initial data $\left(u_{0}, \varphi_{0}, p_{0}\right)^{\top}$, the equilibrium are the constant states $(u, \varphi, p)=$ $\left(\omega_{0}^{2} p_{0}, 0, p_{0}\right)^{\top}$ which belong to $\operatorname{ker}(\mathbf{S})$. The matrix $\mathbf{S}$ is diagonalizable, except for one value of $\varepsilon$ (2.9). Using a basis diagonalizing $\mathbf{S}$ or the Jordan normal form, one writes $\mathbf{U}_{0}=\mathbf{U}_{1}+\mathbf{U}_{2}$ where $\mathbf{U}_{1}$ belongs to $\operatorname{ker}(\mathbf{S})$ and $\mathbf{U}_{2}$ belongs to the plane $P$ in the range of $\mathbf{S}$. Two cases occur:

1. the dissipative case $\varepsilon>0$. Then $\mathbf{U}(t) \rightarrow \mathbf{U}_{1}$ exponentially when $t \rightarrow+\infty$.

2. the conservative case $\varepsilon=0$. Then $\mathbf{U}(t)-\mathbf{U}_{1}$ is an harmonic oscillator in the plane $P$.

4.3. BV estimate for the full system. Now, we are able to obtain uniform estimates of $\mathbf{U}$ on any strip $[0, T] \times \mathbb{R}$ for any positive time $T$. Using the decrease of the norm and of the total variation in each part of the splitting, it is tempting to expect the same decay for the whole splitting scheme, but it is wrong. The reason is that the norms involved in the two steps of the splitting are not the same. For the Burgers part of the splitting, the norm $\max \left(\|u\|_{\infty},\|\varphi\|_{\infty},\|p\|_{\infty}\right)$ is not increasing, and for the ODE part this is the norm $\|U\|_{\infty}$ related to the Euclidean norm (4.8) which is non increasing. The following norm and total variation, well adapted to the Burgers' part of the splitting scheme, are now introduced:

$$
\begin{aligned}
& |\mathbf{U}|=\max (|u|,|\varphi|,|p|), \\
& \|\mathbf{U}\|_{\infty}=\max \left(\|u\|_{\infty},\|\varphi\|_{\infty},\|p\|_{\infty}\right), \\
& T V^{s}(\mathbf{U})=\max \left(T V^{s}(u), T V^{s}(\varphi), T V^{s}(p)\right) .
\end{aligned}
$$


Based on these norms, the following proposition provides bounds sufficient to prove the convergence, as done in $[10,6]$.

Proposition $4.5\left(L^{\infty}\right.$ and $\mathrm{BV}^{s}$ estimates on $\left.(0, T) \times \mathbb{R}\right)$. There exists a positive constant $c$ such that for all $n$ such that $n \Delta t \leq T$,

$$
\begin{aligned}
& \left\|\mathbf{U}^{n}\right\|_{\infty} \leq\left\|\mathbf{U}^{0}\right\|_{\infty} \exp (c T), \\
& T V^{s}\left(\mathbf{U}^{n}\right) \leq T V^{s}\left(\mathbf{U}^{0}\right) \exp (c T) .
\end{aligned}
$$

Proof. The two parts of the splitting are studied successively, by induction. First, solving the Burgers equation and thanks to the maximum principle, one gets $\left\|u^{n+1 / 2}\right\|_{\infty} \leq\left\|u^{n}\right\|_{\infty}$. Morerover $\varphi^{n+1 / 2}=\varphi^{n}, p^{n+1 / 2}=p^{n}$ are unchanged, so that $\left\|\mathbf{U}^{n+1 / 2}\right\|_{\infty} \leq\left\|\mathbf{U}^{n}\right\|_{\infty}$. Second, solving exactly the differential linear system (4.6) can increase the $L^{\infty}$ norm. Let $c>0$ be the matrix norm of $\mathbf{S}$ related to the vectorial norm $\|\cdot\|_{\infty}$ :

$$
c=\sup _{|\mathbf{U}|=1}|\mathbf{S} \mathbf{U}|=\|\mid \mathbf{S}\|_{\infty}
$$

then

$$
\left|\mathbf{U}^{n+1}(x)\right| \leq \exp (c \Delta t)\left|\mathbf{U}^{n+1 / 2}(x)\right| \leq \exp (c \Delta t)\left\|\mathbf{U}^{n+1 / 2}\right\|_{\infty},
$$

and hence:

$$
\left\|\mathbf{U}^{n+1}\right\|_{\infty} \leq \exp (c \Delta t)\left\|\mathbf{U}^{n+1 / 2}\right\|_{\infty} \leq \exp (c \Delta t)\left\|\mathbf{U}^{n}\right\|_{\infty}
$$

This is enough to get the $L^{\infty}$ bound on $[0, T]$.

The $T V$ bound is obtained in the same way. The total variation decays for the entropy solution of the Burgers equation and the variables $\left(\varphi^{n}, p^{n}\right)$ are unchanged, so

$$
T V^{s}\left(\mathbf{U}^{n+1 / 2}\right) \leq T V^{s}\left(\mathbf{U}^{n}\right)
$$

The differential system (4.6) is linear, thus $\mathbf{V}_{i}^{n+1}=\mathbf{U}^{n+1}\left(x_{i+1}\right)-\mathbf{U}^{n+1}\left(x_{i}\right)$ satisfies the same system and

$$
\left|\mathbf{U}^{n+1}\left(x_{i+1}\right)-\mathbf{U}^{n+1}\left(x_{i}\right)\right| \leq \exp (c \Delta t)\left|\mathbf{U}^{n+1 / 2}\left(x_{i+1}\right)-\mathbf{U}^{n+1 / 2}\left(x_{i}\right)\right| .
$$

Adding on $i$ and taking the supremum yields

$$
T V^{s}\left(\mathbf{U}^{n+1}\right) \leq \exp (c \Delta t) T V^{s}\left(\mathbf{U}^{n+1 / 2}\right) \leq \exp (c \Delta t) T V^{s}\left(\mathbf{U}^{n}\right),
$$

which concludes the proof.

These BV bounds provide the compactness in space, and hence the compactness in space and time through a bound in $\operatorname{Lip}_{t} L_{x}^{1}$. Then, passing to the limit, the same bound is obtained for an entropy solution. The passage to the limit is classic, see for instance $[17,27,28,34]$. In the case of fractional $\mathrm{BV}^{s}$, the Lipschitz estimate in time is replaced by a Hölder estimate $[5,6,12]$.

4.4. Uniqueness with only Burgers' entropies. The uniqueness is a consequence of two stability results w.r.t the $L^{1}$ norm: first, the Kruzkov stability for a scalar equation with a source term, second, the stability of the solutions of a differential system. The general case for weakly coupled system is discussed in Sec. 5. In the 
particular case of the system (2.8), let us consider two initial data $\mathbf{U}_{0}$ and $\widetilde{\mathbf{U}}_{0}$ and the corresponding solutions $\mathbf{U}$ and $\widetilde{\mathbf{U}}$.

Using the entropies only for the first equation (2.4a), the Kruzkov method of doubling of variables $[18,10]$ yields for a scalar equation with a source term:

$$
\int_{\mathbb{R}}|u-\widetilde{u}|(x, t) d x \leq \int_{\mathbb{R}}\left|u_{0}-\widetilde{u}_{0}\right|(x) d x+\int_{0}^{T} \int_{\mathbb{R}} \Omega^{2}|\varphi-\widetilde{\varphi}|(x, t) d x d t .
$$

Next, substracting $\varphi$ and $\widetilde{\varphi}$ and also $p$ and $\widetilde{p}$ in the two last equations of the linear system (2.4b)-(2.4c), we have directly for an explicit constant $C>0$ :

$$
\begin{aligned}
\int_{\mathbb{R}}(|\varphi-\widetilde{\varphi}|+ & |p-\widetilde{p}|)(x, t) d x \leq \int_{\mathbb{R}}\left(\left|\varphi_{0}-\widetilde{\varphi}_{0}\right|+\left|p_{0}-\widetilde{p}_{0}\right|\right)(x, t) d x \\
& +C \int_{0}^{T} \int_{\mathbb{R}}(|u-\widetilde{u}|+|\varphi-\widetilde{\varphi}|+|p-\widetilde{p}|)(x, t) d x d t .
\end{aligned}
$$

The two inequalities are added. Gronwall lemma provides the $L^{1}$ stability.

4.5. Energy inequality. Now, we turn to prove inequality (4.4).

Proof. Let $e=\frac{1}{2}\left(u^{2}+\Omega^{2} \varphi^{2}+\Omega^{2} \omega_{0}^{2} p^{2}\right)$ be the density of energy and $E=\int_{\mathbb{R}} e d x$ be the total energy. The latter is written

$$
E^{n+1}-E^{n}=\underbrace{E^{n+1 / 2}-E^{n}}_{I_{1}}+\underbrace{E^{n+1}-E^{n+1 / 2}}_{I_{2}} .
$$

During the first part of the splitting, the entropy inequality for the exact solution of the Burgers equation satisfies in the sense of distributions

$$
\partial_{t} u^{2}+\partial_{x} u^{3} / 6 \leq 0, \quad u \in \mathcal{D}^{\prime}
$$

By integration in space, one obtains $\int_{\mathbb{R}}\left(u^{n+1 / 2}\right)^{2} d x \leq \int_{\mathbb{R}}\left(u^{n}\right)^{2} d x$. Since $\varphi$ and $p$ are constant during this step, it follows $I_{1} \leq 0$. During the second part of the splitting, the solution of the differential system (4.7) satisfies

$$
\partial_{t} e=-\varepsilon \Omega^{2} \varphi^{2}
$$

Integrating the latter equation over $\left[t_{n}, t_{n+1}\right]$ and approximating the r.h.s. yields

$$
e^{n+1}-e^{n+1 / 2}=-\int_{t_{n}}^{t_{n}+\Delta t} \varepsilon \Omega^{2} \varphi^{2} d t \leq-\Delta t \varepsilon \Omega^{2}\left(\varphi^{n+1 / 2}\right)^{2}+\mathcal{O}(\Delta t)^{2} .
$$

Integration in space provides an inequality about $I_{2}$. Summing the contributions of $I_{1}$ and $I_{2}$ gives

$$
\frac{E^{n+1}-E^{n}}{\Delta t} \leq-\int_{\mathbb{R}} \varepsilon \Omega^{2}\left(\varphi^{n+1 / 2}\right)^{2} d x+\mathcal{O}(\Delta t) .
$$

Passing to the limit, the inequality (4.4) is obtained. 
5. Weakly coupled systems in $\mathbf{B V}^{s}$. The system (2.4) studied along this paper is a particular case of weakly coupled hyperbolic systems:

$$
\left\{\begin{array}{l}
\partial_{t} u_{i}+\partial_{x} f_{i}\left(u_{i}\right)=g_{i}(\mathbf{U}), \\
\mathbf{U}(x, 0)=\mathbf{U}_{0}(x)
\end{array}\right.
$$

where $i=1, \ldots, d, \mathbf{U}=\left(u_{1}, \ldots, u_{d}\right)^{\top}, \mathbf{G}(\mathbf{U})=\left(g_{1}(\mathbf{U}), \ldots, g_{d}(\mathbf{U})\right)^{\top}$ is a Lipschitz function and $\mathbf{U}_{0} \in L^{\infty}$. In the case of system (2.4), there is only one nonlinear flux $f_{1}\left(u_{1}\right)=u_{1}^{2} / 2, f_{i} \equiv 0$ for $i>1$, and a linear source term.

Classical results about weakly coupled systems in $L^{\infty}$ are recalled. Then a new result about the propagation of $\mathrm{BV}^{s}$ regularity will be proven.

Definition 5.1 (Entropy solutions [32]). A function $\mathbf{U}$ in

$$
L_{l o c}^{\infty}\left(\mathbb{R} \times\left[0,+\infty\left[, \mathbb{R}^{d}\right) \cup C^{0}\left(\left[0,+\infty\left[, L_{l o c}^{1}\left(\mathbb{R}, \mathbb{R}^{d}\right)\right.\right.\right.\right.\right.
$$

is said to be an entropy solution of $(5.1 \mathrm{~b})$ with initial data $\mathbf{U}_{0}=\left(u_{i}^{0}, \cdots, u_{d}^{0}\right)^{\top}$ if for all diagonal convex entropy $\eta(\mathbf{U})=\left(\eta_{1}\left(u_{1}\right), \ldots, \eta_{d}\left(u_{d}\right)\right)^{\top}, \eta_{i}$ convex for all $i$, and for all diagonal nonnegative smooth function $\phi$ with compact support in $\mathbb{R} \times[0,+\infty[$, $\phi=\left(\phi_{1}, \ldots, \phi_{d}\right)^{\top}, \phi_{i}(x, t) \geq 0$, we have for all $i=1, \ldots, d$ :

$$
\begin{aligned}
& \int_{0}^{+\infty} \int_{\mathbb{R}}\left(\eta_{i}\left(u_{i}\right) \partial_{t} \phi_{i}+q_{i}\left(u_{i}\right) \partial_{x} \phi_{i}+\eta^{\prime}(\mathbf{U}) g_{i}(\mathbf{U}) \phi\right) d x d t \\
& +\int_{\mathbb{R}} \eta_{i}\left(u_{i}^{0}(x)\right) \phi_{i}(x, 0) d x \geq 0,
\end{aligned}
$$

where $\mathbf{q}=\left(q_{1}, \cdots, q_{d}\right)^{\top}$ is the diagonal entropy-flux, with $q_{i}^{\prime}=\eta_{i}^{\prime} f_{i}^{\prime}$.

THEOREM 5.2 (Existence and uniqueness for weakly coupled system [32]). The system $(5.1 \mathrm{~b})$ with the initial data $\mathbf{U}_{0} \in L^{\infty}\left(\mathbb{R}, \mathbb{R}^{d}\right)$ has one and only one entropy solution $\mathbf{U} \in C^{0}\left(\left[0,+\infty\left[, L_{\text {loc }}^{1}\left(\mathbb{R}, \mathbb{R}^{d}\right)\right)\right.\right.$.

The reader is refered to [32] for the proof. The global existence is simply a consequence of the global Lipschitz assumption on G. In the case of system (2.4), weaker conditions than those of Theorem 5.2 were needed to prove existence and uniqueness of the entropy solution. In Sec. 4, Theorem 4.3 has been proven indeed with only one family of entropy.

TheOREM 5.3 ( $\mathrm{BV}^{s}$ entropy solution). If the initial data $\mathbf{U}_{0}$ belongs to $B V^{s}\left(\mathbb{R}, \mathbb{R}^{d}\right)$ for $0<s \leq 1$, then the unique entropy solution $\mathbf{U}$ of (5.1b) belongs to

$$
L_{\text {loc }}^{\infty}\left(\left[0,+\infty\left[, B V^{s}\left(\mathbb{R}, \mathbb{R}^{d}\right)\right) \cap \operatorname{Lip}_{\text {loc }}^{s}\left(\left[0,+\infty\left[, L_{\text {loc }}^{1 / s}\left(\mathbb{R}, \mathbb{R}^{d}\right)\right) .\right.\right.\right.\right.
$$

Proof. We follow the same lines than for the proof of Theorem 4.3. The Lipschitz regularity of $\mathbf{G}$ ensures linear estimates. Again, a splitting scheme is used. Let us explain on the first small interval of time $[0, \Delta t]$ how to proceed:

1. $\mathbf{U}^{1 / 2}$ is the exact solution of the $d$ decoupled scalar conservation laws:

$$
\left\{\begin{array}{l}
\partial_{t} u_{i}+\partial_{x} f_{i}\left(u_{i}\right)=0, \\
u_{i}(x, 0)=u_{i}^{0}(x),
\end{array} \quad i=1, \ldots, d .\right.
$$

2. $\mathbf{U}^{1}$ is the exact solution of the nonlinear ODE:

$$
\left\{\begin{array}{l}
\partial_{t} \mathbf{U}=\mathbf{G}(\mathbf{U}), \\
\mathbf{U}(x, 0)=\mathbf{U}^{1 / 2}(x) .
\end{array}\right.
$$


The only point is to check that, for some constant $c>0$,

$$
T V^{s}\left(\mathbf{U}^{1}(., \Delta t)\right) \leq \exp (c \Delta t) T V^{s}\left(\mathbf{U}^{1 / 2}(., \Delta t)\right) \leq \exp (c \Delta t) T V^{s}\left(\mathbf{U}^{0}(., 0)\right) .
$$

For the first inequality, the Lipschitz stability w.r.t. to the initial data is used. Let $x<y$ be fixed, then for some $c>0$ depending only on the G Lipschitz norm, one has:

$$
\left|\mathbf{U}^{1}(x, \Delta t)-\mathbf{U}^{1}(y, \Delta t)\right| \leq \exp (c \Delta t)\left|\mathbf{U}^{1}(x, 0)-\mathbf{U}^{1}(y, 0)\right| .
$$

Thus $T V^{s}\left(\mathbf{U}^{1}(., \Delta t)\right) \leq \exp (c p \Delta t) T V^{s}\left(\mathbf{U}^{1}(., 0)\right)$ with $p=1 / s$. To conclude the first inequality of (5.5), one notices the equality $\mathbf{U}^{1}(., 0)=\mathbf{U}^{1 / 2}(., \Delta t)$. The second inequality of (5.5) is simply the $T V^{s}$ decay of all conservation laws [5]. Iterating the argument concludes the proof.

6. Conclusion. Some questions remain open and deserve further mathematical investigations:

- Is only one strictly convex entropy $\eta$ sufficient to caracterize the unique entropy solution of system (1.1)? This result has been proven for the first time by Panov [33] for homogeneous conservation laws with a convex flux.

- The inequality of energy (4.4) can be understood as an entropy inequality. This entropy does not depend only on $u$ but on the three components. Is the energy inequality enough to caracterize the unique entropy solution of system $(1.1)$ ?

- The dissipative term in (1.1b) is purely phenomenological and it only accounts for losses in the resonators. A more detailed modeling is needed to lead to a quantitative agreement between simulations and experiments [35]

$$
\left\{\begin{array}{l}
\partial_{t} u+\partial_{x}\left(a u+b \frac{u^{2}}{2}\right)=c \partial_{t}^{-1 / 2} \partial_{x} u-\Omega^{2} \partial_{t} p \\
\partial_{t}^{2} p+\varepsilon \partial_{t}^{3 / 2} p+\omega_{0}^{2} p-m \partial_{t}^{2} p^{2}+n\left|\partial_{t} p\right| \partial_{t} p=u
\end{array}\right.
$$

The visco-thermal losses in the tube and in the resonators are modeled by fractional derivatives in time (coefficients $c$ and $\varepsilon$ ). The nonlinear attenuation at the neck of the resonators is also taken into account (coefficient $n$ ). The analysis of (6.1) requires further efforts.

Acknowledgments. This research was supported by the GdR MecaWave, CNRS, France. Much of the work of the first author was performed while visiting the Laboratoire de Mécanique et d'Acoustique in 2018, supported by the CNRS. The authors are grateful to Marie Touboul for a careful reading of the manuscript.

\section{REFERENCES}

[1] K. Beauchard and E. Zuazua, Large time asymptotics for partially dissipative hyperbolic systems, Arch. Ration. Mech. Anal., 199-1 (2011), 177-227.

[2] C. Bellis And B. Lombard, Simulating transient wave phenomena in acoustic metamaterials using auxiliary fields, Wave Motion, 86 (2019), 175-194.

[3] H. Berjamin, N. Favrie, B. Lombard and G. Chiavassa, Nonlinear waves in solids with slow dynamics: an internal variable model, Proc. R. Soc. Lond. A Math. Phys. Eng. Sci., 473 (2017), 20170024.

[4] H. Berjamin, B. Lombard, G. Chiavassa and N. Favrie, A finite-volume approach to $1 D$ nonlinear elastic waves: application to slow dynamics, Acta Acustica United with Acustica, 104 (2018), 561-570. 
[5] C. Bourdarias, M. Gisclon and S. Junca, Fractional BV spaces and first applications to conservation laws, J. Hyperbolic Differ. Equ., 11-4 (2014), 655-677.

[6] C. Bourdarias, M. Gisclon, S. Junca, Y.J. Peng, Eulerian and Lagrangian formulations in $B V^{s}$ for gas-solid chromatography, Commun. Math. Sci., 14-6 (2016), 1665-1685.

[7] A. Bressan and K.T. NGuyen, Global existence of weak solutions for the Burgers-Hilbert equation, SIAM J. Math. Anal., 46-4 (2014), 2884-2904.

[8] A. Chaigne and J. Kergomard, Acoustics of Musical Instruments, Springer-Verlag (2016).

[9] F.H. Clarke, Y.S. Ledyaev, R.J. Stern and P.R. Wolenski, Nonsmooth Analysis and Control Theory, Graduate Texts in Mathematics 178, Springer (1998).

[10] C.M. Dafermos, Hyperbolic Conservation Laws in Continuum Physics, Springer Verlag, BerlinHeidelberg (2016).

[11] C. De Lellis, F. Otto And M. Westdickenberg, Structure of entropy solutions for multidimensional scalar conservation laws, Arch. Ration. Mech. Anal., 170-2 (2003), 137-184.

[12] B. Guelmame, S. Junca And D. Clamond, Regularizing effect for conservation laws with a Lipschitz convex flux, to appear in Communications in Mathematical Sciences (2020).

[13] M.R. Haberman And A.N. Norris, Acoustic metamaterials, Acoustics Today, 12-3 (2016), 31-39.

[14] M. F. Hamilton and D. T. Blackstock, Nonlinear Acoustics, Academic Press (1998).

[15] B. Hanouzet And R. NATAlini, Global existence of smooth solutions for partially dissipative hyperbolic systems with a convex entropy, Arch. Ration. Mech. Anal., 169-2 (2003), 89-117.

[16] J. H. Hubbard and B. H. West, Differential Equations: a Dynamical System Approach, Texts in Applied Mathematics (Springer-Verlag, 1991).

[17] S. Junca And M. RAscle, Strong relaxation of the isothermal Euler system to the heat equation, Z. Angew. Math. Phys., 53-2 (2002), 239-264.

[18] S. N. Kruzkov, First order quasilinear equations with several independent variables, Mat. Sb., 81-123 (1970), 228255.

[19] P.D. LAX, Hyperbolic systems of conservation laws II, Comm. Pure Appl. Math., 10 (1957) 537-566.

[20] P.D. Lax, Hyperbolic Partial Differential Equations, Courant Lecture Notes in Mathematics 14, American Mathematical Society (2006).

[21] R. J. LeVeque, Finite Volume Methods for Hyperbolic Problems, Cambridge Texts in Applied Mathematics, 2002

[22] J. Li And C.T. Chan, Double-negative acoustic metamaterial, Phys. Rev. E, 70 (2004), 055602.

[23] P.-L. Lions, B. Perthame And E. Tadmor, A kinetic formulation of multidimensional scalar conservation laws and related equations, J. Amer. Math. Soc., 7 (1994) 169-192.

[24] B. Lombard And D. Matignon, Diffusive approximation of a time-fractional Burgers equation in nonlinear acoustics, SIAM J. Appl. Math., 76-5 (2016), 1765-1791.

[25] B. Lombard And J.F. Mercier, Numerical modeling of nonlinear acoustic waves in a tube connected with Helmholtz resonators, J. Comput. Phys., 259 (2014), 421-443.

[26] A. MAJDA, Compressible Fluid Flow and Systems of Conservation Laws in Several Space Variables, Springer (1984).

[27] P. Marcati AND R. NATALini, Weak solutions to a hydrodynamic model for semiconductors and relaxation to the drift-diffusion equation, Arch. Ration. Mech. Anal., 129-2 (1995), 129-145.

[28] P. Marcati And R. NAtalini, Weak solutions to a hydrodynamic model for semiconductors: The Cauchy problem, Proc. R. Soc. Edinb. A, 125-1 (1995), 115-131.

[29] E. Marconi, Regularity estimates for scalar conservation laws in one space dimension, J. Hyperbolic Differ. Eq., 15-4 (2018), 623-691.

[30] C. Mascia And R. NATALini, On Relaxation hyperbolic systems violating the ShizutaKawashima condition, Arch. Ration. Mech. Anal. 195-3 (2010), 729-762.

[31] J.F. Mercier And B. LOMBARD, A two-way model for nonlinear acoustic waves in a nonuniform lattice of Helmholtz resonators, Wave Motion, 72 (2017), 260-275.

[32] R. NAtalini And B. Hanouzet, Weakly coupled systems of quasilinear boundary value problem with applications to semilinear elliptic equations, Differ. Integral Equ., 9-6 (1996), 12791292.

[33] E.Y. PANOv, Uniqueness of the solution of the Cauchy problem for a first-order quasilinear equation with an admissible strictly convex entropy, Mat. Zametki, 55-5 (1994), 116-129.

[34] F. Poupaud, M. Rascle And J.P. Vila, Global solutions to the isothermal Euler-Poisson system with arbitrarily large data, J. Differ. Equations, 123-1 (1995), 93-121.

[35] O. Richoux, B. Lombard And J.F. Mercier, Generation of acoustic solitary waves in a lattice of Helmholtz resonators, Wave Motion 56 (2015), 85-99.

[36] Y. Shizuta And S. KaWASHima, Systems of equations of hyperbolic-parabolic type with application to discrete Boltzmann equation, Hokkaido Math. J., 14 (1985), 249-275. 
[37] N. Sugimoto, Propagation of nonlinear acoustic waves in a tunnel with an array of Helmholtz resonators, J. Fluid. Mech., 244 (1992), 55-78.

[38] N. Sugimoto, Acoustic solitary waves in a tunnel with an array of Helmholtz resonators, J. Acoust. Soc. Am., 99-4 (1996) 1971-1976.

[39] N. Sugimoto, M. Masuda, J. Ohno And D. Motoi, Experimental demonstration of generation and propagation of acoustic solitary waves in a air-filled tube, Phys. Rev. Lett., 83-20 (1999) 4053-4056.

[40] N. Sugimoto, M. Masuda, K. Yamashita and H. Horimoto, Verification of acoustic solitary waves, J. Fluid. Mech., 504 (2004) 271-299.

[41] W.A. Yong, Entropy and global existence for hyperbolic balance laws, Arch. Ration. Mech. Anal., 172-2 (2004), 247266.

[42] Y. ZENG, Gas dynamics in thermal nonequilibrium and general hyperbolic systems with relaxation, Arch. Ration. Mech. Anal. 150-3 (1999), 225279. 